\title{
La Convention interminable : les régicides au tribunal du passé (1815-1830)
}

The interminable Convention : the regicides before the tribunal of the past

(1815-1830)

\section{Côme Simien}

\section{OpenEdition}

\section{Journals}

Édition électronique

URL : https://journals.openedition.org/ahrf/13637

DOI : 10.4000/ahrf.13637

ISSN : 1952-403X

Éditeur :

Armand Colin, Société des études robespierristes

Édition imprimée

Date de publication : 1 septembre 2015

Pagination : 189-211

ISBN : 9782200929855

ISSN : 0003-4436

\section{Référence électronique}

Côme Simien, « La Convention interminable : les régicides au tribunal du passé (1815-1830) », Annales

historiques de la Révolution française [En ligne], 381 | juillet-septembre 2015, mis en ligne le 01

septembre 2018, consulté le 23 avril 2022. URL : http://journals.openedition.org/ahrf/13637 ; DOI :

https://doi.org/10.4000/ahrf.13637 


\title{
LA CONVENTION INTERMINABLE : LES RÉGICIDES AU TRIBUNAL DU PASSÉ (1815-1830)
}

Côme SIMIEN

\begin{abstract}
La loi d'amnistie du 12 janvier 1816, qui exclut à perpétuité du territoire national les conventionnels régicides qui se sont ralliés aux Cent-Jours, engage les autorités de la Restauration, en quelques semaines, dans un important travail d'investigation historique et mémorielle sur le passé révolutionnaire des anciens députés de la Convention. Ceux-ci, par ailleurs soumis, désormais, à une surveillance permanente, se retrouvent non seulement prisonniers du passé, mais encore prisonniers de la politique (toutes leurs actions, tous leurs déplacements, toutes leurs fréquentations sont analysés au prisme du complot politique). Les implications de cette "pathologie mémorielle » de la Restauration, qui affecte aussi bien les députés condamnés à l'exil que ceux qui bénéficient a priori du pardon royal, sont loin de n'être que théoriques. Le présent article se propose donc d'interroger les formes, les sens et les conséquences de cette impossible mémoire apaisée de la Convention, en analysant plus particulièrement les trajectoires des anciens députés de l'Ariège, de l'Aude et des Pyrénées-Orientales de 1815 à 1830.
\end{abstract}

Mots clés : régicides, conventionnels, mémoire de la Révolution, Restauration

«L'ombre du régicide plane sur toute la Restauration », rappelait récemment Bettina Frederking ${ }^{1}$. Avec des nuances chronologiques, toutefois. La première Restauration (avril 1814-mars 1815) s'était efforcée de

(1) Bettina FrEDERKING, « La condamnation des régicides en France sous la Restauration, entre culpabilité collective et conflit mémoriel », dans Paul CHOPELIN et Sylvène ÉDOUARD (dir.), $L e$ sang des princes. Cultes et mémoires des souverains suppliciés, $X V I^{e}-X X I^{e}$ siècle, Rennes, PUR, 2014, p. 115 . 
dissocier l'acte $d u$ régicide de la personne des régicides ${ }^{2}$. Un temps, le régime de Louis XVIII crut ainsi pouvoir tenir ensemble les deux bouts opposés d'une mémoire affrontée, en sacralisant la mémoire du « roimartyr », garante de sa légitimité restaurée, tout en respectant, dans le même temps, la promesse de pardon faite par Louis XVI à ses ennemis, dans son testament. Conformément aux dernières volontés de son frère, Louis XVIII place donc au cœur de la Charte, un «pacte de pardon et d'oubli $»^{3}$. Dès son préambule, celle-ci fixe « avec netteté, le principe de damnatio memoriae $»^{4}:$ «Nous avons effacé de notre souvenir, comme nous voudrions qu'on pût les effacer de l'histoire, tous les maux qui ont affligé la patrie durant notre absence ». Son article 11 précise : "Toutes recherches des opinions et votes émis jusqu'à la Restauration sont interdites. Le même oubli est commandé aux tribunaux et aux citoyens ». Les deux mémoires - voire les deux nations - issues de la Révolution, devaient ainsi réussir à coexister dans une même communauté nationale ${ }^{5}$. En théorie du moins, car dès 1814-1815, la mémoire sacrificielle et volontiers vengeresse des partisans les plus outrés de la Restauration n'a de cesse de contredire le dogme d'État du pardon ${ }^{6}$.

L'expérience des Cent-Jours (mars-juin 1815) rompt irrémédiablement ces équilibres précaires. Les trois mois du printemps 1815 ont en effet été vécus comme un véritable traumatisme par toute une génération, pour qui le retour de Napoléon n'est pas un simple coup d'État (à la différence du 18 Brumaire), mais un véritable mouvement de subversion sociale. Pour les contemporains, avec les Cent-Jours, c'est « la Révolution, assoupie pendant quinze ans [qui] recommence $»^{7}$. Ce retour en force d'une « Révolution imaginée, voire imaginaire ${ }^{8}$ provoque une " recharge contre-révolutionnaire du régime $»^{9}$. Aussi, lorsqu'en juillet 1815 Louis XVIII est réinstallé sur le trône, le pardon et l'oubli ne sont désormais

(2) Emmanuel FUREIX, « Regards sur le(s) régicide(s), 1814-1830. Restauration et recharge contre-révolutionnaire », Siècles, n²3, 2006, p. 32.

(3) Raymond HUARD, «Les conventionnels "régicides" après 1815 : aperçu historiographique et données historiques », dans Roger BOURDERON (dir.), Saint-Denis ou le jugement dernier des rois, Saint-Denis, Éditions PSD, 1993, p. 289.

(4) Emmanuel FUREIX, «Regards sur le(s) régicide(s)... », op. cit., p. 33.

(5) Ibidem.

(6) Bettina FREDERKING, op. cit., p. 117.

(7) Emmanuel de WARESQUIEL, L'Histoire à rebrousse-poil. Les élites, la Restauration, la Révolution, Paris, Fayard, 2005, p. 151-152.

(8) Ibidem.

(9) Emmanuel FUREIX, « Regards sur le(s) régicide(s)... », op. cit., p. 32. 
plus d'époque. L'heure est au contraire à l'expiation de ce passé révolutionnaire revenu trop brutalement hanter le présent ${ }^{10}$. Le 11 novembre 1815 , les ultras, dominants à l'Assemblée ${ }^{11}$, lancent l'assaut contre les anciens conventionnels, par l'intermédiaire de La Bourdonnaye. Selon eux, le crime dont les régicides se sont rendus coupables vingt-trois ans plus tôt les place au-dehors de la nation. Leur bannissement relèverait d'une mesure de sûreté générale : les régicides, et surtout ceux qui ont adhéré aux Cent-Jours, sont des individus dangereux ${ }^{12}$. Anciens révolutionnaires, régicides et, pour nombre d'entre eux, soutiens de l'Empire, ils cristallisent les figures de l'opposition au régime. Tout concourt donc à faire de leur exil une pressante nécessité politique. Le 12 janvier 1816, la Chambre adopte une loi «d'amnistie » pour ceux qui ont soutenu le retour de Bonaparte. Son article 7, imposé par les ultra-royalistes, fixe une limite claire entre ceux qui pourront ou non bénéficier du pardon royal. Plus précisément, cet article ordonne l'exclusion à perpétuité du territoire national des conventionnels régicides ayant adhéré à l'Acte additionnel ou accepté un emploi du gouvernement des Cent-Jours. Il les prive de leur patrie, de leurs droits civils et de la faculté d'y posséder aucun bien, titre ou pension ${ }^{13}$. Au gré d'une interprétation extensive de la notion de « régicide » et de l'adhésion aux Cent-Jours ${ }^{14}, 84 \%$ des régicides survivants (soit un groupe d'un peu plus de deux cents individus), déclarés « ennemis irréconciliables de la France et du Gouvernement légitime », doivent, dans un délai d'un mois, prendre le chemin de l'exil.

Dans le sillage des travaux récents sur l'étude du régicide ${ }^{15}$, nous nous proposons d'interroger les formes et les sens pris par son impossible mémoire apaisée à l'échelle d'une vie, celle des anciens conventionnels, dans cet intervalle de temps - pour eux hostile - ouvert par la loi d'amnistie de 1816 et refermé par la Révolution de juillet 1830, qui permet aux exilés survivants de réintégrer la nation française. La volumineuse correspondance administrative suscitée par la loi d'amnistie du 12 janvier 1816 permet d'esquisser quelques réponses sur ce point particulier. Nous

(10) Voir Idem, La France des larmes. Deuils politiques à l'âge romantique (1814-1840), Seyssel, Champ-Vallon, 2009.

(11) Francis DÉMIER, La France de la Restauration (1814-1830). L'impossible retour du passé, Paris, Gallimard, 2012, p. 187-188.

(12) Raymond HUARD, op. cit., p. 290-291.

(13) Bettina FREDERKING, op. cit., p. 119.

(14) Voir Raymond HuARD, op. cit., p. 283-299.

(15) Cf. les trois articles déjà cités de Raymond HuARD (1993), Emmanuel FuREIX (2006) et Bettina Frederking (2014), ou encore Mona OzOUF, « Ballanche : l'idée et l'image du régicide », dans Idem, L'homme nouveau. Essais sur la Révolution française, Paris, Gallimard, 1989, p. 183-210. 
resserrerons l'étude sur les ex-conventionnels de trois départements méridionaux (l'Ariège, l'Aude et les Pyrénées-Orientales), avec une attention plus particulière pour le cas ariégeois, où les échanges entre le ministre de la Police générale, le préfet, les sous-préfets et maires ont été minutieusement conservés ${ }^{16}$.

\section{Prisonniers du passé : la Convention nationale comme histoire imposée d'une vie}

Pour que la loi du 12 janvier 1816 puisse être appliquée à tous les régicides encore en vie, le ministre de la Police générale, Élie Decazes, engage, au lendemain de l'adoption de la loi, un important travail d'investigation, impliquant toute la chaîne d'autorité, des services du ministère aux préfets, puis des sous-préfets aux maires, afin d'opérer le tri entre ceux des conventionnels qui furent régicides et ceux qui ne le furent pas, de s'assurer que les premiers sont toujours en vie, de les localiser, puis d'écarter du groupe des régicides ceux qui n'auraient rempli aucune fonction publique durant les Cents-Jours ni signé l'Acte additionnel ${ }^{17}$. Dès le 13 janvier 1816, Decazes adresse à cette fin une circulaire à tous les préfets du royaume ${ }^{18}$. Par celle-ci, il les enjoint de lui faire parvenir, dans les meilleurs délais, « le Tableau général de toutes les personnes de cette classe [les régicides] qui se trouveraient dans votre département », en précisant que ce tableau devra mentionner « leur âge, leur fortune, la famille à laquelle elles appartiennent, l'influence qu'elles pourraient conserver, les fonctions qu'elles ont remplies pendant la Révolution, et particulièrement après le retour de l'usurpateur ; la conduite enfin qu'elles auraient tenue, à diverses époques ».

Ainsi sommées d'enfreindre le principe de damnatio memoriae fixé par la Charte, les autorités locales vont répondre avec une étonnante célérité. Il est vrai que les luttes d'influences qui travaillent la seconde Restauration sont aussi des luttes entre groupes de mémoires affrontées ${ }^{19}$. Tout le monde trouve alors son compte dans cette enquête si sensible. D'une part, depuis

(16) Ces documents, réunis par Albert Tournier, ne sont pas inconnus des historiens locaux, qui ont déjà été amenés à les exploiter au début du $\mathrm{XX}^{\mathrm{e}}$ siècle. Voir notamment Jean Gros, « Les conventionnels de l'Ariège en 1816 », Revue des Pyrénées, t. XIX, 1907.

(17) La députation de l'Ariège, de l'Aude et des Pyrénées-Orientales à la Convention nationale fut composée de 20 conventionnels. 14 sont considérés comme régicides en 1816 , avec des nuances sensibles d'un département à l'autre : 6 sur 6 en Ariège, 5 sur 8 dans l'Aude, 3 sur 6 dans les Pyrénées-Orientales. janvier 1816.

(18) AN, $\mathrm{F}^{7} 6707$, Circulaire du ministre de la Police générale aux préfets du royaume, 13

(19) Emmanuel FurEIX, La France des larmes..., op. cit., p. 138. 
l'été 1815, une importante épuration du corps préfectoral a fait la part belle aux ultras, c'est-à-dire aux principaux artisans de l'exclusion de la nation des régicides et aux promoteurs d'une mémoire vengeresse légitimant une justice punitive tournée contre les anciens révolutionnaires ${ }^{20}$. Il n'est donc guère surprenant de les voir agir promptement, à l'instar de Claude-Joseph Trouvée ${ }^{21}$, préfet de l'Aude, qui transmet au ministre de la Police générale un tableau détaillé des régicides résidant dans son département dès le 21 février ${ }^{22}$. Mais un préfet comme celui de l'Ariège, qui ne se rattache pas au courant ultra-royaliste est plus prompt encore à réagir. Préfet d'Empire depuis 1810, le baron Chassepot de Chapelaine fut suspendu lors de la première Restauration. Il est néanmoins réinstallé dans le département de l'Ariège en août 1815, à la place du préfet provisoire nommé à l'issue des Cent-Jours par le duc d'Angoulême. Cette nomination est impopulaire au sein du milieu ultra et la position du baron de Chapelaine demeure instable tant que l'ultra-royaliste Vaublanc occupe le ministère de l'Intérieur ${ }^{23}$. L'enquête du ministre de la Police générale, Decazes, un royaliste modéré engagé, au même moment, dans une vigoureuse concurrence avec le ministre de l'Intérieur pour le contrôle de l'information ${ }^{24}$, lui offre sans doute un moyen de raffermir tant sa position vis-à-vis de l'État monarchique (en jouant la Police générale et la modération contre l'Intérieur et l'ultraroyalisme), que son autorité à l'égard de ses sous-préfets, lesquels lui ont été imposés par les Ultras. D’où le zèle qu'il déploie au cours du premier semestre 1816 : il n'adresse pas moins de trente-cinq lettres, entre fin janvier et septembre 1816, au sujet des régicides de son département (il n'en reste pourtant plus que deux sur six), à ses sous-préfets, au ministre de la Police générale, mais aussi aux préfets des Basses-Alpes, des Pyrénées-Orientales, de la Haute-Garonne et de la Dordogne ${ }^{25}$. Dès le 23 janvier, il transmet à Decazes de premiers éléments d'information ${ }^{26}$. Le même jour, il adresse à ses sous-préfets un tableau reprenant les questions auxquelles le ministre de la Police générale attend des réponses ${ }^{27}$. Trois jours plus tard, le sous-préfet

(20) Voir Nicholas RICHARDSON, The French Prefectoral Corps : 1814-1830, Cambridge, Cambridge University Press, 1966. Et Emmanuel FuREIX, La France des larmes..., op. cit., p. 138.

(21) Ses sympathies ultras sont connues, Nicholas RICHARDSON, op. cit., p 68.

(22) $\mathrm{AN}, \mathrm{F}^{7}$ 6709, Etat des personnes qui habitaient le département de l'Aude et qui ont été atteints [sic] par l'article 7 de la loi d'amnistie du 12 janvier 1816, 21 février 1816.

(23) Nicholas RICHARDSON, op. cit., p. 96.

(24) Pierre KARILA-COHEN, L'État des esprits. L'invention de l'enquête politique en France. 1814-1848, Rennes, PUR, 2008, p. 172.

(25) Pièces de correspondance conservées aux AD 09, 1 J 304.

(26) $\mathrm{AD}$ Ariège, $1 \mathrm{~J} 304$, Lettre du préfet de l'Ariège au ministre de la Police générale, 23 janvier 1816 .

(27) Ibidem, Lettre du sous-préfet de Pamiers au préfet de l'Ariège, 26 janvier 1816. 
de Pamiers lui renvoie le document dûment rempli ${ }^{28}$. Même le sous-préfet d'un arrondissement ne comptant pourtant plus aucun régicide, celui de Foix, se plie à l'exercice, adressant à son supérieur des renseignements détaillés sur les anciens conventionnels Gaston et Lakanal, lesquels ne vivent pourtant plus ici depuis de nombreuses années ${ }^{29}$. Le 7 février, le préfet peut renvoyer au ministère le tableau complet des informations réclamées par Decazes, rassemblées à l'échelle départementale ${ }^{30}$.

L'enquête Decazes ravive ainsi, en quelques semaines, non seulement la mémoire de la Révolution, dans sa globalité, mais aussi les mots, les concepts, les catégories politiques, les discours et les postures du passé, soudainement re-convoquées dans le présent. Dans ces tableaux sous-préfectoraux puis préfectoraux, les informations renvoyant à l'avantRévolution sont en effet réduites à leur portion congrue. De Vadier, par exemple, nous saurons seulement qu'il était fils de domestique et qu'il fut « conseiller au Sénéchal de Pamiers $»^{31}$. De Lakanal et de Raymond Gaston qu'ils exerçaient les fonctions de « professeur $»^{32}$. Les sous-préfets s'arrêtent en revanche beaucoup plus longuement sur la période révolutionnaire. Cela passe tout d'abord par un rappel des fonctions électives occupées par les régicides de l'aube de la Révolution au crépuscule de la Convention. Vadier fut ainsi «nommé député à l'Assemblée constituante. À son retour, président du tribunal du district, puis conventionnel et membre du comité de Sûreté générale ». Le professeur Gaston, lui, « fut élu Juge de paix au commencement de la Révolution, il manifesta dès le principe les opinions les plus exagérées ; nommé vers la fin de 1792 membre de la Convention ». Une fois rappelée leur élection à l'Assemblée nationale en septembre 1792, le propos s'enrichit de considérations morales et révèle la mémoire locale encore vive des moments traumatiques de la Révolution, lesquels sont articulés aux faits les plus saillants de la carrière révolutionnaire des ex-conventionnels dont il s'agit de rendre compte. Vadier se

(28) Ibid., Lettre du sous-préfet de Pamiers au préfet de l'Ariège, 22 janvier 1816.

(29) Ibid., État sur la position actuelle, la conduite morale et politique des individus de l'arrondissement de Foix qui ont assisté ou concouru à l'horrible procès de Louis XVI comme membre de la Convention, s.d.

(30) Ibid., Lettre du préfet de l'Ariège au ministre de la Police générale, 15 février 1816. Ce tableau est conservé aux AN, $\mathrm{F}^{7}$ 6709, Renseignements sur les individus du département de l'Ariège qui ont été à la Convention nationale et ont concouru au procès de Louis XVI, s.d.

(31) Ibid., État des habitants de l'arrondissement de Pamiers qui ont concouru au procès de Louis XVI, dressé par le sous-préfet de Pamiers, 26 janvier 1816.

(32) Ibid., État sur la position actuelle, la conduite morale et politique des individus de l'arrondissement de Foix qui ont assisté ou concouru à l'horrible procès de Louis XVI comme membre de la Convention, s.d. 
fit ainsi « remarquer dans l'Assemblée constituante par un discours où il appeloit le roi un Brigand couronné ». De ce mot fameux prononcé par le député ariégeois à l'été 1791, suite à la fuite à Varennes, la présentation passe ensuite directement à la " Terreur », temps d'autant mieux remémoré à Pamiers que l'attitude de Vadier à cette époque eut des incidences sur les élites ariégeoises : " Il se montra le bourreau de son pays en faisant périr sur l'échaffaud quatorze notables ». Mais, « après la mort de Robespiere [sic], Vadier fut condamné à la déportation. Enfin, il s'étoit retiré dans son domaine ». Gaston, à la Convention, « se fit connaître par les motions les plus incendiaires et les plus extravagantes. Jacobin exalté, il fut envoyé en mission dans les départements et près des armées, et se signala par des actes de férocité. Il siégea constamment au haut de la Montagne ; il vota la mort de Louis XVI sans restriction ». Itinéraire somme toute proche de celui de Lakanal, lequel se montra à la Convention « ardent partisan des principes de la Montagne et du nivellement, il y brûla ses lettres de prêtrise, fut envoyé en mission comme propagandiste, dans les départements du Nord ; il concourut à la formation du calendrier républicain. Il a voté la mort du roi sans restriction ». Parce que Lakanal est passé en mission par la Dordogne, le préfet de ce département, à la demande de son collègue de l'Ariège, fait ce même travail de mémoire locale, réduisant volontiers l'action révolutionnaire du régicide à la sauvagerie la plus élémentaire :

«Il y fut dans les temps affreux de la Révolution envoyé comme représentant pour établir une manufacture d'armes. [...] Mais il ne borna pas là sa mission ; comme un vandale il fit détruire plusieurs châteaux, dépouilla quelques autres de leurs plus beaux ornements de peintures et de librairie, proclama les principes les plus immoraux, qu'il s'efforça de justifier par son exemple ; et puis rentra à la Convention au commencement de $1795 »^{33}$.

Enfin, les sous-préfets donnent quelques indications sur l'itinéraire des régicides au lendemain de la séparation de la Convention. Lakanal, « réélu membre du conseil des Cinq-Cents », occupa ensuite, «sous le règne de l'usurpateur », différentes fonctions dans « l'éducation publique ». Vadier ne sortit de son domaine qu'après « le retour de l'usurpateur pour aller à Toulouse où il est prétendu qu'il se réunit aux fédérés ». Dans ces tableaux, l'attitude adoptée par les ex-conventionnels lors du procès de Louis XVI tend à perdre en centralité. Il est symptomatique, à cet égard, 
que le sous-préfet de Pamiers, arrivé à la fin de son analyse historique de la vie en Révolution du régicide Vadier, rajoute in extremis ces derniers mots : « Je ne dois pas oublier de dire qu'il assista au jugement de Louis XVI et qu'il vota la mort ». À l'heure de réécrire la vie des révolutionnaires, c'est le moment de la Convention nationale qui tend à envahir l'essentiel du champ chronologique. C'est lui qui condamne véritablement ceux de ses membres qui sont encore vivants. C'est lui aussi qui perd la mémoire de ceux qui sont déjà morts.

À Paris, un travail complémentaire est accompli par les services du ministère de la Police générale : des notices sont rédigées pour chacun des régicides, qu'ils soient encore vivants ou qu'ils soient déjà morts. Non liées aux tableaux remontés des départements, ces notices sont historiques dans leur démarche : après que la liste des régicides a été dressée par le ministère avec l'aide du conservateur des $\operatorname{archives}^{34}$, le résumé de leur existence révolutionnaire semble bien avoir été composé, lui aussi, à l'aide des archives laissées par les Assemblées nationales de la décennie 1790. Inégalement fournies selon la masse des informations qu'il a été possible de réunir sur les prises de parole de tel ou tel conventionnel (la notice d'Azéma s'achève ainsi rapidement après l'évocation du procès de Louis XVI, parce que le député de l'Aude « ne parut plus à la tribune $\left.{ }^{35}\right)$, ces biographies s'étirent sur quelques lignes à peine pour les plus courtes $\left(\right.$ Montégut $^{36}$, Espert $^{37}$, mais sur deux pages pour les plus longues (Birotteau ${ }^{38}$ et Clauzel $^{39}$, pourtant morts). Aussi peu loquaces que les tableaux préfectoraux sur la vie prérévolutionnaire des régicides, elles rappellent en revanche presque immédiatement la position adoptée par les députés lors du procès du roi. Alors que les notices auraient dû s'en tenir là, puis passer au printemps 1815 pour mettre à jour un possible ralliement des régicides à « l'Usurpateur », elles n'évoquent ni les Cent-Jours ni la période impériale. Le récit historique, souvent plus descriptif que partisan, se concentre sur la période révolutionnaire au cours de laquelle le régicide fut représentant du peuple. Cherchant à situer les députés dans les clivages politiques de l'époque (qui des girondins, tel Birotteau ; qui des montagnards tels Espert,

(34) $\mathrm{AN} \mathrm{F}^{7} 6707$, Discussion interprétative et historique des mesures prises pour l'application de la loi d'amnistie du 12 janvier 1816.

(35) AN, $F^{7}$ 6710, Notice sur Michel Azéma, s.d.

(36) $\mathrm{AN}, \mathrm{F}^{7} 6714$, Notice sur François-Étienne-Sébastien Montégut, s.d.

(37) AN, $\mathrm{F}^{7}$ 6712, Notice sur Jean Espert, s.d.

(38) AN, $F^{7} 6710$, Notice sur Jean-Baptiste Birotteau, s.d.

(39) Ibidem, Notice sur Jean-Baptiste Clauzel, s.d. 
Cassanyès $^{40}$, ou des jacobins tels Vadier ${ }^{41}$, Azéma qui « appuya toutes leurs mesures »...), ces notices révèlent une forte attention aux combats entre les factions révolutionnaires, en cherchant à mettre au jour le rôle des uns et des autres lors de la « révolution du 31 mai » 1793 (Cassanyès « contribua à la chute des Girondins »), du 9 Thermidor (dans laquelle Vadier joua un rôle actif), lors des procès contre les thermidoriens (Bonnet, l'un des membres chargés d'examiner la conduite de Carrier, «se prononça franchement contre lui $»^{42}$; tandis que Clauzel se «prononça avec véhémence contre Collot, Billaud et Barère »), ou lors des journées de germinal et prairial an III (Girard s'y «prononça contre la faction terroriste ${ }^{43}$ ). En outre, ces notices rappellent tant les attitudes des conventionnels, avec force détails (Espert qui siégeait « à côté de Robespierre »; Clauzel qui devant la «populace qui remplissait les tribunes » lors des journées de Prairial, découvrit sa poitrine aux émeutiers tout en leur déclarant « que ceux qui voulaient remplacer les représentants du peuple en marchant sur leurs cadavres ne travailleraient pas avec plus de zèle à son salut »), que leurs discours, allant jusqu'à citer certains d'entre eux - certes pas tous, loin s'en faut. Ainsi est-il noté que lors du procès de Louis XVI, Girard, « député de l'Aude à la Convention nationale, dit, en votant pour l'appel au peuple : «Il est enfin arrivé ce jour de vengeance d'une faction infâme. Français, consolez vous ; il est arrivé aussi le jour du triomphe de l'humanité !». La notice de Clauzel rappelle encore sa prise de position, après Thermidor, contre l'abrogation de la loi du 17 septembre 1793 : « Il ne faut pas que les aristocrates pensent que la Convention veuille les protéger, parce qu'elle a mis l'humanité à l'ordre du jour ». Ajoutons que l'on n'y retrace pas seulement les faits les plus compromettants ou les prises de parole les plus exaltées. La notice de Marragon ${ }^{44}$ rappelle par exemple qu'il « travailla beaucoup dans les comités d'Agriculture et de Travaux publics, et présenta des plans sur les moyens de vivifier la navigation intérieure ». Le propos, enfin, ne s'interrompt pas en brumaire an IV, avec la séparation de la Convention. Il continue au contraire de décrire les prises de position des ex-conventionnels, avec le même degré de précision, tant que ceux-ci demeurent législateurs révolutionnaires, soit au sein du conseil des CinqCents, soit au sein du conseil des Anciens. La notice de Cassanyès s'achève

(40) Ibid., Notice sur Jacques-Joseph-François Cassanyès, s.d.

(41) AN, $F^{7} 6715$, Notice sur Marc-Guillaume-Alexis Vadier, s.d

(42) AN, $F^{7}$ 6710, Notice sur Pierre-François-Dominique Bonnet, s.d.

(43) AN, $F^{7}$ 6712, Notice sur Antoine Girard, s.d.

(44) AN, $F^{7} 6714$, Notice sur Jean-Baptiste Marragon, s.d. 
ainsi par ces mots : il est « sorti du corps législatif le 20 mai 1797. Depuis lors il n'y est plus reparu ». Et pour ceux qui continuèrent d'être législateurs après Brumaire, le coup d'État de Bonaparte met malgré tout un terme à la notice, à l'image de celle de Clauzel qui, après avoir précisé que ce dernier « sortit du corps législatif en mai 1798 », ajoute ensuite que « la Révolution du 18 Brumaire le ramena au corps législatif ». Il mourut en $1804 »$.

En interrompant brutalement la progression du récit biographique des ex-conventionnels à leur sortie de la représentation nationale, la distance temporelle entre le présent monarchique et le passé de l'engagement révolutionnaire des conventionnels se trouve symboliquement aboli, l'un et l'autre étant englobés « dans un seul et même horizon historique ${ }^{45}$. Le face à face organisé de la sorte avec un passé qui ne passe pas ne définit toutefois pas la seule identité administrative des anciens conventionnels. Il n'est pas qu'une manière d'organiser la trame de leur vie dans les bureaux des sous-préfectures et des ministères. Il a également des répercussions sur leur existence quotidienne. Dès novembre 1815, alors que s'engagent les discussions sur la loi d'amnistie à la Chambre, le sous-préfet de l'arrondissement de Pamiers ne sait quelle position adopter lorsque Jean Espert sollicite un passeport pour se rendre à Rambouillet puis au Havre, pour ses affaires personnelles. Le fonctionnaire se demande en effet s'il n'y aurait pas « des formalités particulières à remplir en ce qui concerne les passeports des individus qui ont siégé à la Convention nationale et ont voté la mort du roi ». Le préfet lui-même, vers qui le sous-préfet s'est tourné, suspend cette délivrance de passeport et s'en justifie auprès du ministre de la Police générale : "J'ai fondé ma détermination sur la surveillance que vous me prescrivez d'exercer en pareille matière, et sur l'extrême circonspection que vous me recommandez, et dont on ne saurait trop user à l'égard de certains personnages $»^{46}$. Il faut finalement attendre le mois suivant pour que le ministre en personne donne son accord. C'est la même histoire qui recommence, en 1822, lorsque Jean Espert sollicite, certificat de bonne conduite à l'appui, un passeport pour gagner la Hollande. Début avril, le sous-préfet de Pamiers refuse d'homologuer le certificat par lequel le maire de Roumengoux avait attesté de la bonne conduite et des bonnes vie et mœurs de l'ex-conventionnel. S'en expliquant au préfet, il déclare : «Je n'ai pas besoin de vous dire que la qualité de régicide est la véritable cause

(45) Reinhart KOSELLECK, Le futur passé. Contribution à la sémantique des temps historiques, Paris, Éd. de l'EHESS, 1990, p. 20.

(46) $\mathrm{AN}, \mathrm{F}^{7}$ 6712, Lettre du préfet de l'Ariège au ministre de la Police générale, 22 novembre 1815. 
de mon refus, car le maire en ne spécifiant ni le temps, ni le lieu est censé approuver le crime au moins implicitement $\gg{ }^{47}$. En cela, et contrairement à ce que pouvait écrire Sergio Luzzatto, les conventionnels ont bien le droit au souvenir dans la France de la Restauration, un souvenir officiel omniprésent, obsessionnel, inquiet, minutieux et volontiers tracassier ${ }^{48}$.

Mais à ramener ainsi l'impossible mémoire apaisée de la Convention dans le présent, les autorités rendaient possible la réouverture des blessures non cicatrisées du passé. Comme le faisait récemment remarquer Emmanuel Fureix, la Restauration, en usant et en abusant de la mémoire révolutionnaire, finit par développer une pathologie mémorielle qui ne manque pas de faire resurgir des souvenirs accusateurs ${ }^{49}$. Depuis la deuxième quinzaine du mois de mars 1816, après une visite domiciliaire effectuée dans son domaine du Peyroutet, les autorités préfectorales de l'Ariège sont informées que Vadier est désormais installé à Mons (Belgique). Pourtant, fin mai-début juin 1816, le « sieur Tillagre commandant une poignée de Garde nationale de Montaut » se rend « au domicile du sieur Vadier », de sa seule autorité. Il cherche à y désarmer le sieur Delpat, qui administre les affaires de l'ancien régicide en son absence du royaume. Delpat est invectivé par la petite troupe, ainsi que les autres résidents de la maisonnée du vieux conventionnel ${ }^{50}$. Pourquoi cette expédition vexatoire ? Le préfet de l'Ariège croit savoir « que le sieur Tillagre [doit] la perte d'un frère aux dénonciations de Vadier ». Or, si la pratique de la vengeance se libère au printemps 1816 , c'est bien, selon le préfet, en raison du remue-ménage du passé des conventionnels opéré par les autorités depuis le début de l'année : «Quelque coupable que soit le sieur Vadier et ses adhérents ceci ne donne pas le droit de les maltraiter en parole quand il s'agit d'un acte administratif que les circonstances exigent [la surveillance et l'exil de Vadier], mais qu'il est inutile et déplacé d'accompagner de paroles grossières $»^{51}$.

Et c'est bien parce que les autorités n'ont de cesse de leur reprocher leur passé, dans le cadre de la loi d'amnistie, que les ex-conventionnels se laissent eux-mêmes gagner, en ces temps de Restauration puis d'exil, par cette contagion mémorielle qui ramène tout, inlassablement, à la

(47) AD Ariège, $1 \mathrm{~J}$ 304, Lettre du sous-préfet de Pamiers au préfet de l'Ariège, 4 avril 1822.

(48) Sergio LuZZATTO, Mémoire de la Terreur. Vieux montagnards et jeunes républicains au XIX $X^{e}$ siècle, Lyon, PUL, 1991, p. 70.

(49) Emmanuel FUREIX, La France des larmes..., op. cit., p. 147.

(50) AD Ariège, $1 \mathrm{~J}$ 304, Lettre du préfet de l'Ariège au sous-préfet de Pamiers, 2 juin 1816.

(51) Ibidem. 
Convention $^{52}$. Parmi d'autres possibles, l'exemple de Montegut (PyrénéesOrientales) est révélateur de ce rapport au temps particulier suscité chez les anciens conventionnels par la loi du 12 janvier 1816 : dans une lettre écrite à Boissy d'Anglas, le 18 juin 1819, depuis la Suisse, afin que ce dernier l'aide dans les démarches qu'il a entreprises auprès du ministère pour rentrer en France, il use d'un « cher collègue » riche de nombreux sens. Certes, la formule tient à la dissymétrie de la position des deux hommes. La position de requérant de Montégut le conduit sans doute à mobiliser cette formule qui lui permet de remémorer à Boissy d'Anglas, désormais pair de France, ce qui les lie l'un à l'autre. Il n'en reste pas moins que l'exilé réactualise ainsi la période de la Convention ${ }^{53}$. Faut-il rappeler, encore, que ceux des ex-conventionnels qui rédigent leurs mémoires depuis cet exil qui leur a été imposé n'ont de cesse d'écrire leur "futur au passé », revenant sans fin sur ce moment de leur vie devenu indépassable : les quatre interminables années de la Convention ${ }^{54}$ ?

À l'heure où, à la charnière des XVIII ${ }^{\mathrm{e}}$ et $\mathrm{XIX}^{\mathrm{e}}$ siècles, les sociétés européennes sont peu à peu gagnées par un nouveau rapport au temps, au sein duquel le présent s'affirme progressivement comme un futur à écrire ${ }^{55}$, l'horizon d'attente des ex-conventionnels reste donc, lui, résolument tourné vers le passé ${ }^{56}$. L'ironie est sensible lorsqu'elle touche des hommes qui, au cœur de la décennie révolutionnaire, avaient eu l'ambition d'abolir le passé et d'inventer le futur. Que les conventionnels aient décidé ou non de se retirer des affaires publiques depuis lors importe peu : le rapport au temps qui leur est imposé est bien celui d'un temps statique, figé dans son état révolutionnaire en général, et dans le moment « Convention nationale » (1792-1795) en particulier. À l'image de l'idée républicaine qu'ils ont incarnée, ils se trouvent ainsi placés tout à la fois «dans l'histoire, et hors de l'histoire ${ }^{57}$. Les survivants comme les morts de la première République ne sont donc pas seulement marqués par les «stigmates indélébiles de la république de la Terreur », comme l'écrivait Alan B. Spitzer ${ }^{58}$. Ce sont les stigmates de la Convention toute entière qu'on leur impose de porter, et par élargissement ceux de tout leur passé de législateur, bien au-delà

(52) Sergio LuZZATTO, op. cit., p. 27 et p. 69.

(53) AN, $\mathrm{F}^{7}$ 6714, Lettre de Montégut à Boissy d'Anglas, 18 juin 1819.

(54) Sergio LuZZATTO, op. cit., p. 27 et sq.

(55) François HARTOG, Régimes d'historicité. Présentisme et expériences du temps, Paris, Le Seuil, 2003, p. 77-107.

(56) Pour emprunter les mots de Reinhart KoselLeCK, op. cit., p. 307.

(57) Claude NICOLET, L'idée républicaine en France, Paris, Gallimard, 1982, p. 133.

(58) Alan B. SPITZER, «La République souterraine », dans François FURET et Mona Ozouf (dir.), Le siècle de l'avènement républicain, Paris, Gallimard, 1993, p. 348. 
du seul acte du régicide, dont la place paraît somme toute modeste dans ces réécritures de l'engagement révolutionnaire des ex-conventionnels. En 1815-1816, la Convention n'était ni terminée ni même terminable.

\section{Prisonniers de la politique}

L'abolition de l'écart temporel entre la Révolution et la Restauration faisait des conventionnels davantage que de simples vestiges d'un passé révolu. La « dé-distanciation » scripturaire opérée entre le présent et leurs engagements de la décennie 1790 pouvait conduire les autorités royales à les identifier tout entier à l'aune de ce qu'ils furent autrefois : des militants d'une cause politique révolutionnaire. Cette insistance de l'administration royale à lire et à écrire l'histoire des ex-conventionnels non sous le signe de l'altérité mais du « Même », pour reprendre les analyses de Paul Ricoeur, rendait pensable l'hypothèse d'une « réeffectuation » de leur passé dans le présent ${ }^{59}$. Sans doute, une telle appréhension de la vie des régicides a pu être alimentée par la conduite politique réelle des anciens députés : sur les neuf régicides de l'Ariège, de l'Aude et des Pyrénées-Orientales encore en vie en 1815-1816, six sont condamnés à l'exil par la loi d'amnistie. Six d'entre eux se sont donc ralliés à l'empereur lors des Cent-Jours. Deux (Marragon et Montegut) pour avoir approuvé l'Acte additionnel aux Constitutions de l'Empire. Deux autres pour avoir approuvé celui-ci et occupé des fonctions publiques durant les Cent-Jours (Lakanal membre de l'Institut ; RamelNogaret, préfet du Calvados $\left.{ }^{60}\right)$. Deux, enfin, Vadier ${ }^{61}$ et Cassanyès ${ }^{62}$, pour s'être engagés aux côtés des Fédérés, cette milice citoyenne mêlant anciens républicains, bonapartistes et futurs libéraux dans la lutte en faveur d'un 《 bonapartisme révolutionnaire $»^{63}$. Mais Vadier et Cassanyès sont des cas limites, minoritaires par la radicalité de leurs choix durant les Cent-Jours. Comme eux, ils ne sont qu'une quinzaine de régicides à l'échelle nationale à avoir rejoint les Fédérés ${ }^{64}$. Pourtant, ce sont bien tous les régicides, y compris ceux qui, restés en retrait lors du « vol de l'aigle », ne sont pas

(59) Paul Ricoeur, Temps et récit, t. 3, Le temps raconté, Paris, Le Seuil, 1985, p. 257.

(60) AN, $\mathrm{F}^{7} 6709$, État nominatif des personnes qui habitaient le département de l'Aude et qui ont été atteints [sic] par l'article 7 de la loi d'amnistie du 12 janvier 1816, 21 février 1816.

(61) AD Ariège, $1 \mathrm{~J}$ 304, État des habitants de l'arrondissement de Pamiers qui ont concouru au procès de Louis XVI, dressé par le sous-préfet de Pamiers, s.d. (26 janvier 1816).

(62) $\mathrm{AN}, \mathrm{F}^{7} 6709$, État nominatif des personnes qui habitaient le département de l'Aude et qui ont été atteints [sic] par l'article 7 de la loi d'amnistie du 12 janvier 1816, 21 février 1816.

(63) Robert S. AlEXANDER, Bonapartism and Revolutionary Tradition in France. The Fédérés of 1815, Cambrigde, Cambridge University Press, 1991.

(64) Ibidem, p. 93. 
passibles de la loi d'amnistie, qui se trouvent suspectés d'être prêts à reprendre le combat révolutionnaire. Que l'application des dispositions de la loi d'amnistie relative aux régicides ait été confiée, après une brève hésitation, à Decazes et à sa " haute police » politique plutôt qu'au Garde des sceaux est de ce point de vue significatif ${ }^{65}$. Ce choix revenait de facto à conférer un caractère de factieux en puissance aux ex-conventionnels. Cela explique qu'outre les éléments historiques factuels attendus par le ministre, afin de pouvoir appliquer promptement la loi d'amnistie aux anciens conventionnels, Decazes ait exigé des préfets, dans le même temps, une surveillance vigilante et active des moindres faits et gestes des régicides relaps : "Vous aurez à examiner où [ils] se proposeraient de se réunir, les relations et les correspondances qu' [ils] pourraient laisser derrière [eux], les projets qu' [ils] pourraient concevoir, l'espoir qu' [ils] nourriraient encore ». Et Decazes de suggérer, enfin, l'attitude que les préfets devront adopter pour y parvenir : «Il ne s'agit point de les inquiéter, mais de les observer et de les empêcher, autant qu'une sage prévoyance peut le permettre, de devenir inquiétants $»^{66}$.

De même que les notices biographiques des conventionnels défunts n'avaient qu'un rapport fort éloigné avec la loi d'amnistie, de même la prescription d'une telle surveillance dépasse, et de loin, les principes de la loi du 12 janvier 1816. Il est vrai que ces recommandations n'expriment pas seulement les inquiétudes des autorités à l'égard des régicides, mais participent aussi pour partie du « tournant doctrinal » engagé d'une manière plus générale par la police politique, depuis 1814, afin d'en faire moins une force de coercition - la rupture se veut sensible avec la police impériale mise en place par Fouché -, qu'un instrument de « surveillance » de l'opinion publique capable de quadriller l'ensemble du corps social ${ }^{67}$. À cela près que, dans le cas des régicides, la surveillance cible comme autrefois des opinions particulières, surveillance personnalisée « qui ne vaut que lorsqu' on attribue aux individus une puissance qu'ils n'ont plus ${ }^{68}$. Cela révèle donc bien aussi à quel point, par-delà la question éminemment symbolique du régicide, l'attention et la crainte des autorités de la Restauration restent permanentes à l'égard d'hommes redoutés parce qu'ils maintiennent, par leur seule

(65) AN, $\mathrm{F}^{7} 6707$, Discussion interprétative et historique des mesures prises pour l'application de la loi d'amnistie du 12 janvier 1816, s.d.

(66) Ibidem, Circulaire du ministre de la Police générale aux préfets du royaume, 13 janvier 1816.

(67) Pierre KARILA-COHEN, op. cit., p. 82-86.

(68) Ibidem, p. 82. 
existence, une présence de la Révolution, de son histoire et de son principe subversif en ce monde.

À ce jeu, toutes les autorités départementales sont invitées à lire les moindres faits et gestes des anciens législateurs de la France révolutionnaire à l'aune de leur conduite lors de la décennie 1790, c'est-à-dire sous l'angle exclusif du principe révolutionnaire, donc du politique et de la menace permanente pour le régime. Le cas de Jean-Baptiste Espert est, ici, emblématique. En 1816, il est l'un des trois conventionnels des députations de l'Ariège, de l'Aude et des Pyrénées-Orientales à ne pas être condamné à l'exil. Au mois de mai, cependant, le préfet de l'Ariège finit par être convaincu de l'existence dans son département d'un complot contre la monarchie, au centre duquel se trouverait l'ancien conventionnel. Le 23 mai 1816, le maire de Mirepoix, sur la base d'une dénonciation individuelle et confidentielle, l'a informé que, depuis qu'Espert était rentré de Paris, fin mars, il s'était mis à employer journellement «20, 25, 50 jusqu'à 100 ouvriers à son domaine de la Grande Borde » ${ }^{69}$. Au mois de mai, l'embauche retombe brutalement à cinq ouvriers par jour. Or, ajoute l'édile : «Dans un moment où quelques factieux insensés osent encore faire des tentatives pour troubler l'ordre comme il vient d'arriver à Grenoble, la plus légère circonstance éveille l'attention des personnes dévouées au roi et au gouvernement ». À Grenoble, dans la nuit du 4 au 5 mai 1816, Jean-Paul Didier avait essayé de soulever la ville contre les Bourbons. L'activité ouvrière ralentissant à la Grande Borde au moment même où « l'affaire de Grenoble a été connue dans le païs », l'informateur du maire de Mirepoix en a conclu qu'Espert employait préalablement « tant de monde avec quelques dessein ». Le maire, lui, est toutefois plus circonspect : d'une coïncidence « il semble qu'on ait voulu tirer des conjonctures ». Il ajoute : «Peut être, et je veux le croire, ce n'étoit que pour faire quelque travail qu'il a fini. Je l'ignore ». De lettre en lettre, cependant, le doute s'estompe. Le 26 mai 1816 , le préfet de l'Ariège demande au commandant de la gendarmerie de l'Ariège de trouver « quelque moyen secret et raisonnable » pour connaître les motifs de ces recrutements et savoir si l'interruption des embauches est, oui ou non, liée aux événements grenoblois ${ }^{70}$. Le 28 mai, alors qu'aucun renseignement complémentaire ne lui est parvenu, les termes utilisés par le préfet dans le courrier qu'il adresse au ministre de la Police générale ne laissent plus guère de place au doute :

(69) AD Ariège, 1 J 304, Lettre du maire de Mirepoix au préfet de l'Ariège, 23 mai 1816.

(70) Ibidem, Lettre du préfet de l'Ariège au capitaine de Marquis, 26 mai 1816. 
«Le sieur Expert est un des hommes les plus dangereux de mon département. Quoiqu'il était, dans le temps, désarmé, et que j'ai donné des ordres pour que sa conduite soit scrupuleusement surveillée, il sera très difficile de l'empêcher de faire le mal. C'est un de ces êtres pervertis, incorrigibles qui n'agissent jamais ostensiblement, mais toujours de manière à ne pas se compromettre. Il a été renvoyé qu'avant l'événement de Grenoble, le sieur Expert employait tous les jours depuis 25 jusqu'à 100 ouvriers et que lorsque l'événement fût connu, ce nombre a été réduit à cinq. Il existe de forts soupçons pour croire qu'il n'employait un si grand nombre d'ouvriers que pour corrompre l'esprit public $\gg^{71}$.

Le 31 mai 1816, le rapport adressé par la dixième division militaire, stationnée à Mirepoix, au ministre de la Guerre se fait l'écho des mêmes rumeurs. Le lien est alors explicitement noué entre le passé révolutionnaire d'Espert et les mauvaises intentions qui lui sont prêtées : «Le régicide Espert occupait toujours deux cents ouvriers, cet acte de bienfaisance me paraissait avoir un but caché ; aujourd'hui j'en ai presque la certitude par le renvoi qu'il a fait de tous immédiatement après l'affaire de Grenoble ; cet homme est d'une grande influence dans le pays, à raison d'une grande fortune et de son expérience en révolutions $\gg^{72}$.

Le 31 mai 1816, sur un ordre reçu la veille de son commandant, le brigadier de gendarmerie Monié se rend donc à Roumengoux, afin d'épier le régicide. Déguisé en villageois, il passe la matinée à pêcher avec des habitants du lieu, dans les eaux poissonneuses de l'Hers, qui bordent le domaine de Jean Espert au nord et à l'est. Ces villageois, il s'emploie à les faire parler autant que possible de l'ex-conventionnel ${ }^{73}$. Les renseignements qu'il ramène de cette partie de pêche sont cependant bien loin des informations attendues et redoutées par le préfet. Les habitants de Roumengoux avec lesquels le gendarme a pu échanger lui ont expliqué que la centaine d'ouvriers employés journellement par Espert depuis deux mois « travailloit à faire des fossés dans les champs, d'autres remplissoit à demi lesdits fossés de pierres, et ensuite ils le faisaient couvrir de terre pour égoutter les eaux des champs, et d'autres, à faire des bâtisses, etc. ${ }^{74}$. Les mystères du complot dissipés, restent d'importants travaux agraires, réalisés au retour des beaux jours, alors qu'Espert avait été peu présent sur

(71) Ibid., Lettre du préfet de l'Ariège au ministre de la Police générale, 28 mai 1816.

(72) $\mathrm{AN}, \mathrm{F}^{7}$ 6712, Extrait de différents rapports de la 10 ${ }^{\mathrm{e}}$ Division militaire, 31 mai 1816.

(73) AD Ariège, $1 \mathrm{~J} 304$, Lettre du brigadier Monié au commandant de la gendarmerie royale de l'Ariège, $1^{\text {er }}$ juin 1816 .

(74) Ibidem. 
son domaine les mois précédents... Observé depuis Paris, le péril ne paraît d'ailleurs guère sérieux. Le 8 juin, Decazes s'étonne ainsi de l'inquiétude éprouvée par le préfet : «Le sieur Espert a occupé jusqu'à 100 ouvriers, et cet emploi d'une grande fortune n'a paru à quelques personnes qu'une manœuvre politique. L'administration ne doit y voir que l'avantage de l'industrie et du commerce ; et pourquoi en tarir la source ? $\gg^{75}$. Decazes n'en ordonne pas moins de maintenir une stricte surveillance des démarches, actions et discours de l'ex-conventionnel.

L'affaire rebondit deux mois plus tard, cette fois à l'initiative d'un ministre de la Police générale soudainement beaucoup moins confiant. Decazes a été informé que « des réunions, dont les mauvaises intentions ne sauraient être douteuses, ont eu lieu chez le sieur Espert $\gg^{76}$. Il enjoint donc au préfet de lui rendre un « compte détaillé de la conduite» de cet ex-conventionnel régicide, dans un rappel cinglant au baron Chassepot de Chapelaine des devoirs de haute police qui lui sont assignés, particulièrement à l'égard de ceux que la Convention a marqués de son empreinte indélébile : «Je plaçais cet individu sous votre surveillance spéciale [...]. Vôtre silence sur le sieur Espert m'a dû faire penser [...] que cet exconventionnel conservoit la conduite qui convient à sa situation plus encore qu'à celle de tout autre de vos administrés ». Si le préfet rassure Decazes sur ces réunions, il n'a cependant pas oublié l'histoire suspecte des ouvriers du printemps. Il rappelle donc au ministre que ce n'était « probablement pas charitablement pour contribuer à entretenir l'industrie que le sieur Espert occupait jusqu'à deux cents ouvriers par jour à l'époque où une poignée de factieux ourdissaient des complots dans l'Isère, mais bien parce qu'il aura été instruit de leurs coupables desseins et qu'il voulait se ménager un parti à lui, disponible au besoin $\gg^{77}$. S'il avait été effectivement mu par la volonté de soulager l'humanité souffrante, aurait-il brutalement interrompu ces embauches ? «N'a-t-il pas eu, en prenant cette résolution, l'intention d'augmenter alors le nombre des mécontents ? » Cette fois, le ministre se montre beaucoup plus sensible à ceux des arguments qui, en juin encore, lui paraissaient aucunement recevables : «La seule considération locale qui pouvait militer en faveur de cet individu, celle de ses efforts pour encourager l'industrie et secourir la classe ouvrière [...] se trouve en effet détruite par l'effet de leur renvoi [...]. Il deviendrait même important d'examiner jusqu'à quel point sont fondés le rapprochement des dates et la remarque

(75) Ibid., Lettre du ministre de la Police générale au préfet de l'Ariège, 8 juin 1816.

(76) Ibid., Lettre du ministre de la Police générale au préfet de l'Ariège, 2 août 1816.

(77) Ibid., Lettre du préfet de l'Ariège au ministre de la Police générale, 12 août 1816. 
d'une coïncidence de ces dispositions particulières avec les mouvements de quelques factieux dans l'Isère $\gg^{78}$. Le ministre de la Police générale ordonne donc au baron de Chapelaine de convoquer incessamment Espert, afin de lui faire savoir « que l'œil de la police est ouvert sur ses démarches » et qu'à la première inquiétude le concernant, il sera déplacé dans un autre département. Quelques jours plus tard, le préfet convoque donc le régicide et somme le sous-préfet de Pamiers d'exercer « une surveillance spéciale sur cet ex-conventionnel », dont il souhaite qu' on lui rende compte « tous les huit jours $\gg^{79}$. Au mois de septembre, Espert se présente enfin devant le préfet de l'Ariège. De cette entrevue et de cette « surveillance spéciale », l'ex-conventionnel feint de s'étonner auprès de Decazes. Il sait cependant fort bien quelle en est l'origine : non pas l'emploi massif d'ouvriers, mais son passé de législateur, cette Convention qui continue à l'entraver et à faire de lui, tout à la fois, plus et moins qu'un citoyen ordinaire :

«Il y a vingt ans que je n'ai exercé des fonctions publiques dans l'intérieur ni occupé à l'extérieur d'emploi à la nomination du chef du gouvernement. Je n'ai point été fédéré, et je n'ai reçu aucune faveur de Bonaparte. J'ai 59 ans, des infirmités et rien à gagner à un nouvel ordre des choses [...]. La charte a jeté un voile sur ma qualité d'ex-conventionnel $\gg^{80}$.

Si les régicides non exilés par la loi d'amnistie se trouvent ainsi placés, de fait, en résidence surveillée pour activisme politique supposé, il en va de même de ceux des ex-conventionnels bannis du territoire national, longtemps même après 1816. D'abord installé en Suisse (à Vevey), Joseph Cassanyès parvient, en 1821, à être autorisé à s'installer en Espagne. Dès lors, quoiqu'il soit établi au-delà des frontières françaises, la surveillance du préfet du département des Pyrénées-Orientales à son égard est permanente. Jusqu'en 1827, ce dernier en rend régulièrement compte au ministre de l'Intérieur dans ses bulletins de police. Or, lue et recomposée par le premier fonctionnaire du département, toute la vie espagnole de Cassanyès est, à l'image de celle d'Espert, ramenée à d'interminables machinations révolutionnaires. Lorsque, le 28 avril 1821, le préfet informe le ministre que Cassanyès vient de débarquer à Barcelone et compte s'installer à Figueras (au plus près de la frontière française), il ne manque pas de souligner que le régicide est arrivé « de Naples et se trouvait dans un des

(78) Ibid., Lettre du ministre de la Police générale au préfet de l'Ariège, 22 août 1816.

(79) Ibid., Lettre du préfet de l'Ariège au sous-préfet de Pamiers, 28 août 1816.

(80) Souligné par nous, AN, $F^{7} 6712$, Lettre de Jean-Baptiste Espert au ministre de la Police générale, 21 octobre 1816. 
navires qui ont porté en Espagne quelques-uns des révolutionnaires des Deux-Siciles $\gg{ }^{81}$. Le 27 octobre 1824 , le bulletin de police du préfet des Pyrénées-Orientales indique que Cassanyès réside désormais à l'Escala, mais qu'il compte néanmoins regagner Figueras, où il avait vécu lors des années 1821-1822. Or, note le préfet, «il y était alors fort lié avec les transfuges et prenait part à toutes leurs intrigues $»^{82}$. Même à l'Escala, du reste, « Cassanyès ne fréquente [...] que les ennemis du gouvernement monarchique » espagnol ${ }^{83}$. Le regard porté par les autorités ibériques, au même moment, sur le même régicide, offre pourtant une vision fortement dépolitisée de la vie de ce vieil homme, isolé dans un village côtier de la Méditerranée : «Il résulte des informations reçues que Cassanyès est venu de Barcelone s'établir à l'Escala le 14 octobre [1824], qu'il est d'un âge avancé, qu'il exerce la profession de barbier, qu'on le voit généralement aller seul, et toujours taciturne, que sa conduite est régulière et qu'il a peu d'amis ${ }^{84}$. La mémoire de l'engagement révolutionnaire passé des anciens conventionnels les a donc bien rendus prisonniers de la politique. C'est, au fond, un exemple particulièrement sensible de ce que Paul Veyne nommait la « viscosité » des représentations collectives, qui vivent bien plus longtemps que la réalité des groupes auxquels elles renvoient ${ }^{85}$.

\section{Les échelles d'une vie non-conventionnelle}

Reste à se demander si les anciens conventionnels n'eurent aucun moyen d'échapper à cette invasion de leur identité par le politique. Victor Hugo, dans quelques-unes des pages les plus célèbres des Misérables $(1862)^{86}$, narre la visite pastorale rendue en 1815 par Mgr. Myriel, l'évêque de Digne, au conventionnel G., agonisant et vivant reclus à plus de trois quarts d'heure de la ville, «loin de tout hameau, loin de tout chemin, on ne sait quel repli perdu d'un vallon très sauvage ». Les habitants de Digne évitaient à ce point ce lieu, qu'ils qualifiaient de « maison du bourreau » (G., dit-on, à tort d'ailleurs, aurait été régicide), que le « sentier qui y conduisait avait disparu sous l'herbe ». Vingt ans plus tard, dans

(81) AN, $\mathrm{F}^{7}$ 6711, Extrait d'une lettre du préfet des Pyrénées-Orientales, 28 avril 1821.

(82) Ibidem, Extrait du bulletin de police du préfet des Pyrénées-Orientales, 27 octobre 1824.

(83) Ibid., Lettre du secrétaire-générale de la préfecture des Pyrénées-Orientales au ministre de l'Intérieur, 15 mars 1825.

(84) Ibid., Note envoyée à l'ambassadeur Français en Espagne par le premier secrétaire du roi d'Espagne, s.d. (janvier-février 1825).

(85) Paul VEYnE, Le pain et le cirque. Sociologie historique d'un pluralisme politique, Paris, Le Seuil, 1976.

(86) Victor Hugo, Les Misérables, Paris, Gallimard, coll. « La Pléiade », 2013, p. 38-43 
ses Souvenirs d'enfance et de jeunesse $(1883)^{87}$, Ernest Renan évoque à son tour un vieillard de son village d'antan, sans doute un ancien de la Convention : pauvre, ne parlant plus à personne, on l'appelait «Père Système », parce qu'à ses rares interlocuteurs il exposait toujours ses idées en terme de "système », à la manière du XVIII ${ }^{\mathrm{e}}$ siècle finissant. Ces lignes, écrites longtemps après la disparition des derniers révolutionnaires, ont laissé à la postérité un imaginaire durable de ce que fut, localement, la vie des ex-conventionnels après la Révolution. Une vie de parias, condamnés à l'exil intérieur, que tout ramenait, même au village, au moment capital de la Convention, à la politique et au régicide. C'est en s'appuyant sur ces pages que nombre d'historiens ont tenté, par la suite, de retracer le devenir des anciens révolutionnaires aux lendemains de l'Empire ${ }^{88}$. Un retour aux sources conduit cependant à nuancer ce tableau.

De l'enquête Decazes, il ressort que quatre conventionnels seulement sur les neuf encore vivants de l'Ariège, de l'Aude et des Pyrénées-Orientales, résident dans leur département d'élection en 1816. Ce chiffre masque le fait que, sur les treize régicides qui survécurent à la Convention, trois seulement ne revinrent jamais sur leurs terres d'origine après leur sortie de l'Assemblée nationale (Lakanal, Marragon, Clauzel). Parmi les dix qui rentrèrent chez eux, cinq en repartirent certes régulièrement, mais cinq autres semblent être revenus plus durablement vivre chez eux. Pour quelques-uns d'entre eux, ce retour tient sans doute à la faiblesse de leur patrimoine foncier et financier : pour un Cassanyès ou un Montégut, beaucoup moins fortunés qu'un Vadier ou un Espert, il n'était guère possible de vivre en plusieurs lieux différents. L'explication par le patrimoine n'épuise cependant pas à elle seule la question du repli local de nombre d'ex-conventionnels, qu'il ait été occasionnel ou durable.

Ceux qui rentrent s'installent soit dans leur commune natale, soit dans un lieu situé à moins de $10 \mathrm{~km}$ de celle-ci, c'est-à-dire dans leur " pays », là où les interconnaissances les concernant sont les plus denses. Ils ne sont que deux à s'établir un petit peu plus loin de leur petite patrie d'origine : Campmartin, qui se retire à Saint-Girons, localité certes située à plus de $50 \mathrm{~km}$ de son lieu de naissance, mais dont il fut le maire, en 1790, et où il vivait déjà à la veille de la Révolution ${ }^{89}$; Bonnet, enfin, retiré de toute

(87) Ernest RENAN, Souvenirs d'enfance et de jeunesse, Paris, Calmann-Lévy, 1966, p. 69-72.

(88) Voir par exemple Maurice AGULHON, « Survivants de la Révolution », dans idem, Histoire vagabonde, t. III, La politique en France d'hier à aujourd'hui, Paris, Gallimard, 1996, p. 23-24 ; voir aussi Alan B. SPITZER, op. cit., p. 345-347.

(89) Jacques-Philippe GiBOURY, Dictionnaire des régicides, Paris, Perrin, 1989, p. 73. 
activité politique, à compter de l'Empire, s'installe sur sa propriété près de Conques (Aude), à une trentaine de kilomètres seulement de Limoux dont il était originaire ${ }^{90}$. Dans l'ensemble, les bouleversements politiques ne les ont donc pas coupés de leurs assises familiales et territoriales. Le cas de Vadier, ici, est exemplaire : s'il ne se réinstalle pas à Pamiers, il se stabilise cependant dans le domaine du Peyroutet ${ }^{91}$, à quelques encablures de la sous-préfecture, un domaine familial acquis par son grand-oncle en $1719^{92}$. Notons encore que la plupart se retirent soit dans des villages, soit dans de gros bourgs de 3000 habitants au plus. Seul Girard fait exception, en regagnant Narbonne, commune de quelque 9000 habitants à cette époque ${ }^{93}$. Or, dans les agglomérations de moins de 10000 habitants, comme le rappelaient récemment les historiens des guerres de religion réfléchissant sur les cas de « non Saint-Barthélemy », tout le monde se connaît de près ou de loin ${ }^{94}$. Là, il devenait donc possible pour ces anciens législateurs de redevenir autre chose que de simples conventionnels régicides.

Lorsque le brigadier Monié, le 31 mai 1816, pêche avec les villageois de Roumengoux afin d'obtenir des informations sur Jean Espert, plusieurs d'entre eux déclarent remercier le régicide d'avoir embauché ces centaines d'ouvriers : « Sans lui il y aurait eu beaucoup de monde qui aurait crevé de faim », aurait déclaré l'un d'eux au gendarme. Et c'est bien cela qui compte d'abord aux yeux des habitants. Le hiatus est ici sensible entre le regard porté sur Espert par les autorités, tout entier politique, et celui des villageois qui déclarent ne pas ignorer qu'Espert « fut du parti de Bonaparte, qu'il avait été représentant » et qu'il « signa la mort de Louis XVI », toutes réalités politiques néanmoins reléguées à l'arrière-plan d'une identité locale d'Espert d'abord riche d'un sens économique et social ${ }^{95}$. Vadier est quant à lui présenté par le sous-préfet de Pamiers, en janvier 1816, comme étant « fils d'un domestique de M. de Vertamont ancien évêque de Pamiers, dont les bienfaits l'avaient enrichi $»^{96}$. Version quelque peu

(90) Ibidem, p. 53.

(91) AD Ariège, 1 J 304, Lettre du sous-préfet de Pamiers au préfet de l'Ariège, 15 mars 1816.

(92) Gilles DUSSERT, Vadier, Paris, Imprimerie nationale, 1989, p. 22.

(93) Jacques-Philippe GIBOURY, op. cit., p. 180.

(94) Jérémy FOA, « "Bien unis et paisibles" ? Une "non Saint-Barthélemy” à Chalon-sur-Saône (septembre 1572) », dans Véronique CASTAGNET, Olivier CHRISTIN et Naïma GHERMANI (dir.), Les affrontements religieux en Europe du début du XVI ${ }^{e}$ siècle au milieu du XVII ${ }^{e}$ siècle, Villeneuve-d'Ascq, Presses Universitaires du Septentrion, 2008, p. 225.

(95) AD Ariège, $1 \mathrm{~J} \mathrm{304}$, Lettre du brigadier Monier au capitaine commandant de la gendarmerie royale du département de l'Ariège, $1^{\text {er }}$ juin 1816.

(96) Ibidem, État des habitants de l'arrondissement de Pamiers qui ont concouru au procès de Louis XVI, 26 janvier 1816. 
tronquée, mais ô combien signifiante, de l'histoire familiale de Vadier. Son père, en effet, n'était en rien domestique de l'évêque de Pamiers, Mgr. de Verthamont. Son grand-père et son grand-oncle, Guillaume et Pierre Vadier, en revanche, arrivés de Picardie avec Mgr. de Camps, en 1685, furent bel et bien les serviteurs de cet évêque appaméen de la fin du XVII ${ }^{\mathrm{e}}$ siècle. Restés au service de Mgr de Verthamont lorsque celui-ci fut nommé en 1693, ils étaient en réalité non ses « domestiques » mais ses maitres d'hôtel et les receveurs des décimes du clergé du diocèse. À son tour, le père de Marc-Alexis-Guillaume Vadier exerça cette fonction fiscale qui permit aux Vadier de considérablement s'enrichir, en un demi-siècle. La famille devint alors l'une des plus riches de Pamiers. Mais cette ascension sociale trop soudaine d'étrangers au pays suscite bien des racontars et les Vadier ne parviendront jamais à se faire pleinement accepter par la bourgeoisie appaméenne, qui ne les assimile pas. Le père du conventionnel Vadier, toute sa vie durant, sera ainsi (dis)qualifié de « laquais », de « cuisinier », de «domestique $»^{97}$. En rentrant au Peyroutet une première fois sous l'Empire, puis une seconde fois au lendemain des Cent-Jours, Vadier ne retrouve donc pas seulement les souvenirs locaux des persécutions révolutionnaires dont il aurait été l'instigateur depuis Paris ou la mémoire de son discours sur le «brigand couronné ». Il se réinsère dans un univers local fait de rivalités sociales et de jalousies séculaires, bien antérieures à la Convention. Ni Espert ni Vadier ne sont donc dans leur village des « Monsieur Système » ou des conventionnels G. Là, leur passé de législateur et de régicide n'est qu'une des nombreuses facettes de leur identité. L'échelle locale et le jeu des interconnaissances y bloquent l'intrusion du politique dans leur identité individuelle. $\mathrm{Si}$, au village, ces ex-conventionnels sont certes d'anciens révolutionnaires, ce que nul ne saurait ignorer, ils sont aussi des voisins, des riches ou des pauvres, des employeurs ou les héritiers d'anciens parvenus, des amis ou des ennemis séculaires, autant de déterminants parallèles de leur identité, loin des catégorisations exclusivement politiques et fortement dépersonnalisantes adoptées par les autorités ${ }^{98}$.

Jusqu'en 1815-1816, le jeu d'échelles géographiques permet ainsi aux anciens conventionnels de teinter leur identité personnelle de mille nuances tantôt politiques, tantôt sociales, amicales, familiales et professionnelles. La loi du 12 janvier 1816 et le remue-ménage du passé qui l'accompagne tend au contraire à re-politiser entièrement cette identité.

(97) Gilles DUSSERT, op. cit., p. 17-25. p. $225-226$.

(98) Voir pour une analyse similaire à l'époque de la Saint-Barthélemy, Jérémy FOA, op. cit., 
Seuls leur passage à la Convention et l'acte du régicide justifient désormais la présence des bannis au-delà des frontières, en ces lieux où leur étrangeté ne tient qu'à leur fonction passée de législateur révolutionnaire. Pour ceux, peu nombreux, qui échappent à l'exil, le jeu habile des échelles permet de continuer à atténuer l'omniprésence du passé révolutionnaire dans leur vie, mais un peu moins efficacement désormais, à l'heure où leurs déplacements sont plus étroitement surveillés et entravés. Par sa trajectoire en sens inverse des Espert, Vadier ou Azéma, Raymond Gaston confirme paradoxalement la propension des anciens conventionnels à rechercher l'oubli du passé sur les grands chemins du territoire national ${ }^{99}$. Rentré en l'an IV à Foix, sa commune natale, où il devient commissaire du Directoire exécutif de la municipalité, il en repart en l'an VI, alors que la reprise de son militantisme politique dans le contexte du sursaut néo-jacobin le conduit certes à être élu au Conseil des Cinq-Cents (élection annulée) mais aussi, sans doute, à saturer son identité locale par des éléments politiques par trop clivants. Il s'installe donc dans les Basses-Alpes, où il exerce les fonctions de receveur général du département jusqu'à la chute de Napoléon. Lorsque les Cent-Jours réactivent partout la mémoire révolutionnaire, ce partisan zélé de l'empereur (au dire du préfet de la seconde Restauration), au demeurant auxiliaire local de la police de Fouché ${ }^{100}$, quitte Digne et le petit domaine dont il avait fait l'acquisition. Face à ce nouveau renversement de légitimité politique, il fait le choix de gagner la capitale. C'est là, pour lui, dans la plus grande ville qui soit, que se trouve désormais la possibilité de l'oubli : à Paris, où il vit de 1815 jusqu'à sa mort, même le commissaire de police du quartier des Champs-Élysées éprouve les plus grandes difficultés à obtenir quelques renseignements sur sa conduite. Le conventionnel Gaston a trouvé ici le moyen d'entrer « dans la plus profonde obscurité ${ }^{101}$.

Côme Simien CHEC-Université Blaise Pascal - Clermont 2 5 Avenue Carnot 63000 Clermont-Ferrand come.simien@gmail.com

(99) AD Ariège, $1 \mathrm{~J}$ 304, État des habitants de Foix qui ont assisté ou concouru à l'horrible procès de Louis XVI comme membre de la Convention, s.d.

(100) Ibidem, Lettre du préfet des Basses-Alpes au préfet de l'Ariège, 20 février 1816.

(101) AN, $F^{7}$ 6712, Lettre du préfet de Police au ministre de la Police générale, 3 mai 1816. 